Case Report

\title{
Diagnostic Challenges of Pneumocystis Pneumonia during the COVID-19 Pandemic: A Case of a Young Patient with Ground Glass Opacities and Pulmonary Embolism on Chest CT
}

\author{
William Lim (D), Maham Suhail, and Keith Diaz \\ Department of Internal Medicine, Richmond University Medical Center, Staten Island, New York, NY, USA \\ Correspondence should be addressed to William Lim; dr.williamlim6@gmail.com
}

Received 5 June 2021; Revised 1 July 2021; Accepted 23 July 2021; Published 31 July 2021

Academic Editor: George N. Dalekos

Copyright (c) 2021 William Lim et al. This is an open access article distributed under the Creative Commons Attribution License, which permits unrestricted use, distribution, and reproduction in any medium, provided the original work is properly cited.

\begin{abstract}
The coronavirus disease 2019 (COVID-19) pandemic is wreaking havoc across the globe. This pandemic has given rise to a mindset where physicians tend to neglect other causes of pneumonia, especially if the patient presents with signs and symptoms commonly associated with COVID-19. Herein, we report a case of a young man presenting to the emergency department with common clinical, radiological, and laboratory features of COVID-19 pneumonia such as shortness of breath, hypoxia, pulmonary embolism, elevated D-dimer, and bilateral ground glass opacities on computed tomography of the chest but was later diagnosed with Pneumocystis pneumonia that was treated with appropriate antibiotics and corticosteroids. This case highlights the importance of performing a thorough clinical history and differentiating the clinical and radiological features of COVID-19 pneumonia from pneumonia of other etiologies.
\end{abstract}

\section{Introduction}

Since the first case of COVID-19 was diagnosed in December 2019 , it has rapidly spread across the globe, resulting in the COVID-19 pandemic. Presently, with more than 100 million cases of COVID-19 globally, it is not uncommon for physicians to think of COVID-19 as an initial diagnosis for patients presenting with pneumonia [1]. In this report, we discuss a case of a young adult with unremarkable past medical history presenting to the emergency department with shortness of breath and diarrhea. Computed tomography (CT) of the chest revealed diffuse pulmonary bilateral ground glass opacities and pulmonary embolism. Recognizing the differences in radiological and clinical features of COVID-19 and Pneumocystis pneumonia helped to reach the correct diagnosis and, thus, initiate the appropriate treatment for the patient.

\section{Case Presentation}

A 23-year-old male presented to the emergency department (ED) with a chief complaint of diarrhea for the last one week.
On examination, his vital signs were as follows: temperature, 101.8 F; blood pressure, 125/76 mmHg; pulse rate, 126/min; and respiratory rate, $24 / \mathrm{min}$. His blood oxygen saturation was $85 \%$ at room air and $95 \%$ with $5 \mathrm{~L}$ oxygen via nasal cannula. His body mass index (BMI) was $25.1 \mathrm{~kg} / \mathrm{m}^{2}$. Chest examination revealed decreased breath sounds with bilateral crackles in the lower part of lung fields and normal first and second heart sounds.

Initial laboratory investigations revealed these results: white blood cell count (WBC), 7700/ul with lymphopenia; prothrombin time (PT), 16.6 seconds; international normalized ratio (INR), 1.4; activated partial thromboplastin time (APTT), 38.5 seconds; D-dimer, $3.85 \mathrm{mcg} / \mathrm{mL}$, serum lactate dehydrogenase (LDH), $402 \mathrm{U} / \mathrm{L}$; and C-reactive protein (CRP), $3.63 \mathrm{mg} / \mathrm{dl}$. Arterial blood gas (ABG) analysis showed a $\mathrm{pH}$ of $7.53, \mathrm{paCO}_{2}$ of $33 \mathrm{mmHg}, \mathrm{paO}_{2}$ of $62 \mathrm{mmHg}$, serum $\mathrm{HCO}_{3}{ }^{-}$level of $27.6 \mathrm{mmol} / \mathrm{L}$, and alveolararterial (A-a) oxygen gradient of $46.5 \mathrm{mmHg}$.

A chest X-ray (Figure 1) showed patchy bilateral pulmonary opacities. The patient's clinical presentation of shortness of breath and hypoxia together with elevated 


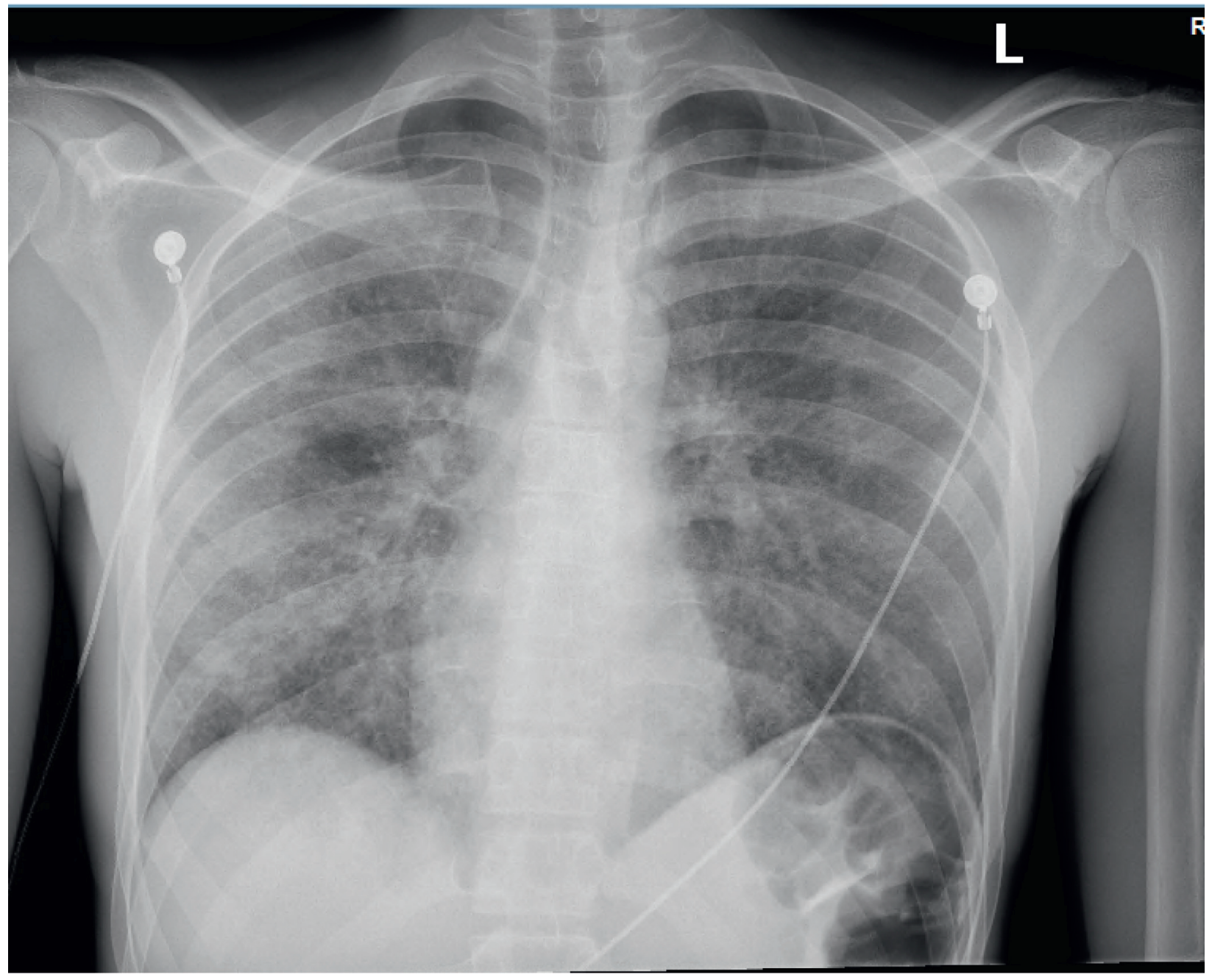

FIgURE 1: CXR showing bilateral pulmonary infiltrates.

D-dimer levels prompted a chest CT to rule out pulmonary embolism. The chest CT demonstrated notable diffuse bilateral ground glass opacities (Figure 2) and arterial embolism in the pulmonary arteries supplying the right middle and lower lobes.

Reverse-transcription polymerase chain reaction (RTPCR) for COVID-19 ribonucleic acid (RNA) conducted on a nasopharyngeal swab sample of the patient revealed a negative result. However, the patient was still considered to have clinical COVID-19, given he had typical features of COVID-19 such as hypoxia, shortness of breath, elevated D-dimer, pulmonary embolism, and ground glass opacities on chest CT. Thereby, dexamethasone $(6 \mathrm{mg})$ for COVID-19 and apixaban for pulmonary embolism were initiated. Decision was made to hold remdesivir since COVID-19 was not confirmed in this patient.

During further questioning, the patient reported productive yellowish cough during the last month coupled with anorexia and significant weight loss of 15 pounds. The insidious onset of the long-lasting symptoms and the radiological features characterized by ground glass opacities seen in Pneumocystis pneumonia as opposed to patchy and peripheral ground glass opacities of typical COVID-19 pneumonia (Figure 3) prompted further investigation for potential occult immunodeficiency. The fourth-generation $\mathrm{Ag} / \mathrm{Ab}$ combination human immunodeficiency virus (HIV)$1 / 2$ immunoassay showed a positive result for HIV-1 antibody and negative for HIV-2 antibody. Repeat PCR tests for COVID-19 on day 3 and day 5 of hospitalization were negative as well. Thereafter, IV trimethoprim-sulfamethoxazole $400 \mathrm{mg}$ every 8 hours was started for possible Pneumocystis pneumonia (PCP) given the new diagnosis of HIV. Dexamethasone was switched to prednisone $40 \mathrm{mg}$ oral twice a day.

Bronchoscopy was performed on day 4 of hospitalization, and bronchoalveolar culture, Gram stain, acid-fast bacilli (AFB) stain, silver stain, and Pneumocystis jirovecii PCR were performed on the specimen obtained. Cells obtained via bronchial washing were negative for malignancy, and no fungal elements were visualized by methenamine silver stain. No acid-fast bacilli were detected on AFB staining, and neither any Mycobacterium species was isolated in the mycobacterial culture. HIV viral load was 1.4 million copies $/ \mathrm{ml}$, and total cluster of differentiation 4 (CD4) count was $<20$ 


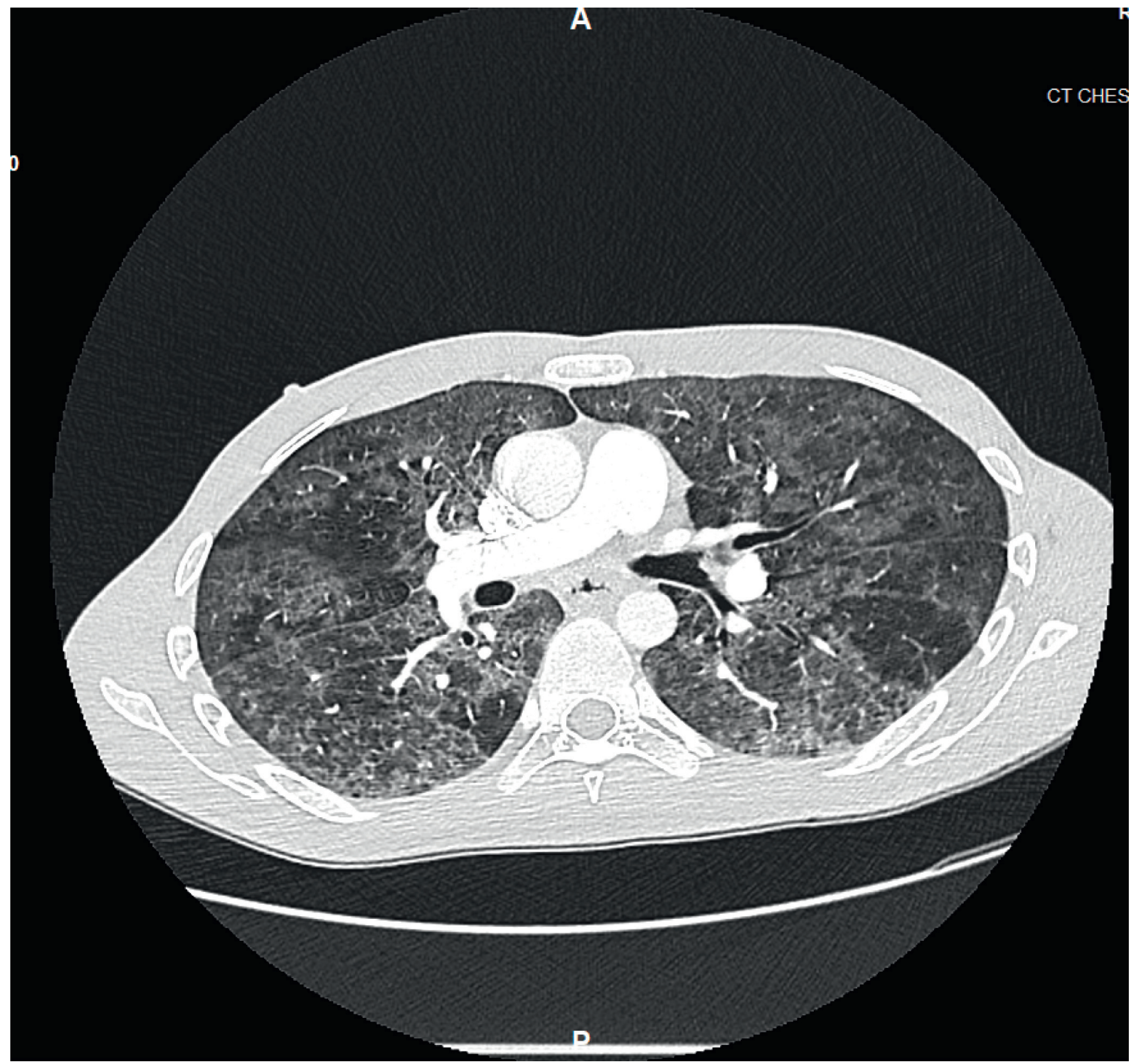

Figure 2: Chest CT showing diffuse bilateral ground glass opacities.

cells/mcl. Treatment with oral emtricitabine/tenofovir and oral dolutegravir was, thus, initiated.

PCR for Pneumocystis jirovecii on material obtained via BAL revealed a positive result. The patient's presentation of chronic symptoms as opposed to acute presentation of COVID-19 patients together with a positive HIV test, high viral load, low CD4 count, and positive PCR for Pneumocystis jirovecii contributed to establishing the diagnosis of Pneumocystis pneumonia. After one more week of continued treatment with IV antibiotics and prednisone, the patient's condition improved and he was discharged with instructions for home oxygen therapy and prescription for apixaban, trimethoprim-sulfamethoxazole $(800 \mathrm{mg} / 160 \mathrm{mg})$, prednisone, dolutegravir, and emtricitabine/tenofovir.

\section{Discussion}

In the current situation of the COVID-19 pandemic, there have been an increasing number of patients presenting with severe symptoms of the disease, such as cough, fever, shortness of breath, hypoxia, and diarrhea. Pneumocystis pneumonia is also characterized by an insidious onset of similar symptoms when found in association with other opportunistic infections. Given these similarities, the diagnosis of Pneumocystis pneumonia may take a backseat in the setting of increasing COVID-19 incidence in the present scenario [2]. In the present case, the patient's presentation of hypoxia, shortness of breath, and diarrhea together with elevated D-dimer and $\mathrm{LDH}$ levels along with pulmonary 


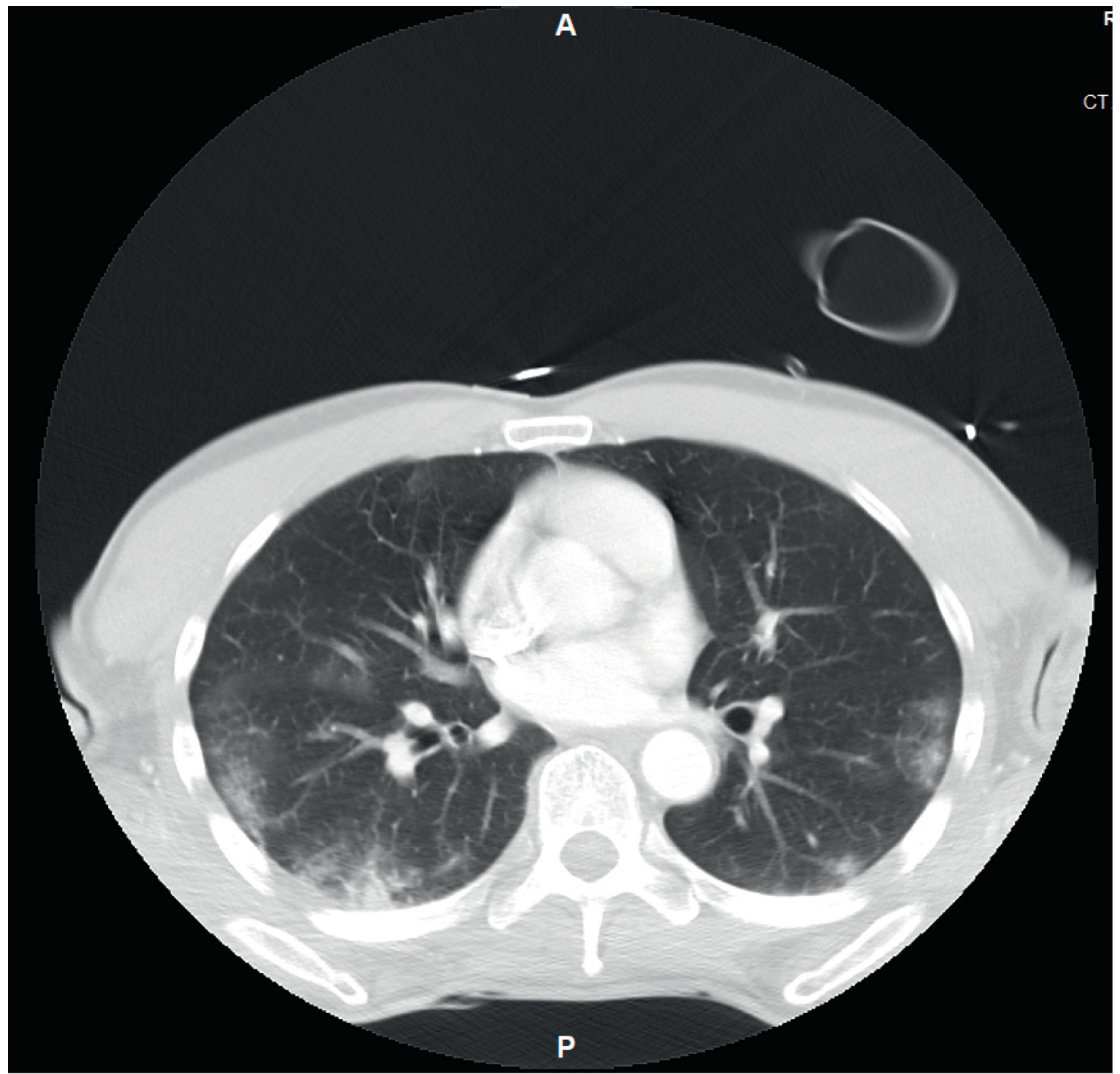

FIgURE 3: Chest CT of the COVID-19 patient showing patchy and peripheral ground glass opacities mid to lower lobe predominant (taken from another COVID-19-positive patient for comparison).

ground glass opacities and pulmonary embolism seen on chest CT made it difficult to differentiate between COVID19 and Pneumocystis pneumonia.

Elevated levels of serum $\mathrm{LDH}$, inflammatory markers (CRP and ferritin), and D-dimer are common laboratory findings associated with COVID-19 [3-5]. Diagnostic tests for COVID-19 include nucleic acid amplification tests (NAAT) such as RT-PCR and antigen testing. RT-PCR assay of COVID-19 RNA from the upper respiratory tract is the preferred initial diagnostic test for COVID-19 with a reported sensitivity of $77 \%[6,7]$.

On the other hand, the common laboratory findings of Pneumocystis pneumonia are low CD-4 counts ( $<200$ cells/ microliter), widened alveolar-arterial oxygen gradient, and elevated LDH level [8-10]. Diagnostic tests for Pneumocystis pneumonia include microscopy with staining and a PCR test. Gomori-methenamine silver is the commonly used stain to detect Pneumocystis pneumonia. The sensitivity and specificity of Grocott-Gomori-methenamine silver stain (GMS) are 79.4 and $99.2 \%$, respectively [11]. PCR has a sensitivity of as high as $96 \%$ and specificity of more than $90 \%$ in detecting Pneumocystis jirovecii in respiratory samples [12-14]. PCR is the most sensitive method for the detection of Pneumocystis and should be considered the diagnostic test of choice for patients with high suspicion of Pneumocystis pneumonia with negative results on GMS stain, as was encountered in the present case [15].

Both COVID-19 and Pneumocystis pneumonia can present with ground glass opacities in the lungs on chest CT, and there are no pathognomonic radiographic findings 
associated with either of the two pathologies that would lead to a definite diagnosis. In general, Pneumocystis pneumonia is characterized by diffuse infiltrates mainly involving the upper lobe while sparing the subpleural regions, whereas COVID-19 is characterized by patchy infiltrates, mainly in the lower lobes with peripheral and subpleural distribution [16-18]. COVID-19 is commonly associated with hypercoagulability which can lead to pulmonary embolism [19-21], whereas pulmonary embolism is not a common feature of Pneumocystis pneumonia [22]. Elevated LDH is a common laboratory abnormality associated with both COVID-19 and Pneumocystis pneumonia, while widened alveolar-arterial oxygen gradient is more commonly found in Pneumocystis pneumonia.

Since the treatment protocols of COVID-19 and Pneumocystis pneumonia are entirely different, it is very important to reach the correct diagnosis. COVID-19 treatment includes remdesevir, baricitinib, or tocilizumab, while the standard regimen for Pneumocystis pneumonia is trimethoprim-sulfamethoxazole for 21 days [23-26]. Corticosteroid of choice used in COVID-19 treatment is dexamethasone with a suggested dosing of $6 \mathrm{mg}$ once daily for 10 days [27-29], while the corticosteroid indicated for moderate-to-severe Pneumocystis pneumonia (partial pressure of oxygen $<70 \mathrm{mmHg}$ at standard room conditions and/or an A-a oxygen gradient of $\geq 35 \mathrm{mmHg}$ ) is prednisone $40 \mathrm{mg}$ twice a day with subsequent taper $[30,31]$. Baricitinib and tocilizumab are immunomodulatory drugs that are indicated for COVID-19 treatment. In the present case, it was of utmost importance to reach the correct diagnosis in order to avoid administration of immunomodulatory agents to an HIV patient with opportunistic infection of Pneumocystis jirovecii, which could have led to deterioration in the patient's condition.

\section{Conclusions}

We report a case of Pneumocystis pneumonia presenting with features mimicking COVID-19 pneumonia. Increased COVID-19 cases in the scenario of the pandemic have led physicians into neglecting other causes of pneumonia and respiratory failure. This case highlights the importance of medical history taking and detailed clinical examination. It also highlights the importance of understanding the differences between clinical and radiological features of COVID-19 and Pneumocystis pneumonia.

\section{Abbreviation}

$\begin{array}{ll}\text { F: } & \text { Fahrenheit } \\ \text { mmHg: } & \text { Millimeter of mercury } \\ \mathrm{min}: & \text { Minute } \\ \mathrm{g} / \mathrm{dl}: & \text { Gram per deciliter } \\ \mathrm{mg} / \mathrm{dl}: & \text { Milligram per deciliter } \\ \mathrm{k} / \mathrm{ul}: & \text { Kilo per microliter } \\ \mathrm{mcg} / \mathrm{mL} \text { FEU: } & \text { Microgram/mL fibrinogen equivalent units } \\ \mathrm{U} / \mathrm{L}: & \text { Unit per liter } \\ \mathrm{mmol} / \mathrm{L}: & \text { Millimole per liter } \\ \mathrm{ml}: & \text { Milliliter } \\ \mathrm{mcl}: & \text { Microliter. }\end{array}$

\section{Data Availability}

No data were used to support this study.

\section{Consent}

No written consent has been obtained from the patients as there are no patient-identifiable data included in this case report.

\section{Conflicts of Interest}

None of the authors have any conflicts of interest.

\section{References}

[1] J. J. Coleman, K. Manavi, E. J. Marson, A. H. Botkai, and E. Sapey, "COVID-19: to be or not to be; that is the diagnostic question," Postgraduate Medical Journal, vol. 96, no. 1137, pp. 392-398, 2020.

[2] S. Mang, D. Kaddu-Mulindwa, C. Metz et al., "Pneumocystis jirovecii pneumonia and severe acute respiratory syndrome coronavirus 2 coinfection in a patient with newly diagnosed HIV-1 infection," Clinical Infectious Diseases, vol. 72, no. 8, pp. 1487-1489, 2021.

[3] F. Zhou, T. Yu, R. Du et al., "Clinical course and risk factors for mortality of adult inpatients with COVID-19 in Wuhan, China: a retrospective cohort study," Lancet, vol. 395, no. 10229, pp. 1054-1062, 2020.

[4] C. Wu, X. Chen, Y. Cai et al., "Risk factors associated with acute respiratory distress syndrome and death in patients with coronavirus disease 2019 pneumonia in Wuhan, China," JAMA Internal Medicine, vol. 180, no. 7, pp. 934-943, 2020.

[5] S. Shi, M. Qin, B. Shen et al., "Association of cardiac injury with mortality in hospitalized patients with COVID-19 in Wuhan, China," JAMA Cardiology, vol. 5, no. 7, pp. 802-810, 2020.

[6] A. Patel and D. B. Jernigan, "Initial public health response and interim clinical guidance for the 2019 novel coronavirus outbreak-United States, december 31, 2019-February 4, 2020," Morbidity and Mortality Weekly Report, vol. 69, no. 5, pp. 140-146, 2020.

[7] B. Clerici, A. Muscatello, F. Bai et al., "Sensitivity of SARSCOVID-2 detection with nasopharyngeal swabs," Front Public Health, vol. 8, Article ID 593491, 2021.

[8] J. D. Stansell, D. H. Osmond, E. Charlebois et al., "Predictors of pneumocystis carinii pneumonia in HIV-infected persons. Pulmonary complications of HIV infection study group," American Journal of Respiratory and Critical Care Medicine, vol. 155, no. 1, pp. 60-66, 1997.

[9] R. F. Miller, L. Huang, and P. D. Walzer, "Pneumocystis pneumonia associated with human immunodeficiency virus," Clinics in Chest Medicine, vol. 34, no. 2, pp. 229-241, 2013.

[10] M. K. Zaman and D. A. White, "Serum lactate dehydrogenase levels and Pneumocystis carinii pneumonia. Diagnostic and prognostic significance," The American Review of Respiratory Disease, vol. 137, no. 4, pp. 796-800, 1988.

[11] G. W. Procop, S. Haddad, J. Quinn et al., "Detection of Pneumocystis jiroveci in respiratory specimens by four staining methods," Journal of Clinical Microbiology, vol. 42, no. 7, pp. 3333-3335, 2004.

[12] M. J. Alvarez-Martinez, J. M. Miro, M. E. Valls et al., "Sensitivity and specificity of nested and real-time PCR for the detection of Pneumocystis jiroveci in clinical specimens," 
Diagnostic Microbiology and Infectious Disease, vol. 56, pp. 153-160, 2006.

[13] P. Flori, B. Bellete, F. Durand et al., "Comparison between real-time PCR, conventional PCR and different staining techniques for diagnosing Pneumocystis jiroveci pneumonia from bronchoalveolar lavage specimens," Journal of Medical Microbiology, vol. 53, no. Pt 7, pp. 603-607, 2004.

[14] A. M. Caliendo, P. L. Hewitt, J. M. Allega, A. Keen, K. L. Ruoff, and M. J. Ferraro, "Performance of a PCR assay for detection of Pneumocystis carinii from respiratory specimens," Journal of Clinical Microbiology, vol. 36, no. 4, pp. 979-982, 1998.

[15] L. Doyle, S. Vogel, and G. W. Procop, "Pneumocystis PCR: it is time to make PCR the test of choice," Open Forum Infectious Diseases, vol. 4, no. 4, Article ID ofx193, 2017.

[16] S. Salehi, A. Abedi, S. Balakrishnan, and A. Gholamrezanezhad, "Coronavirus disease 2019 (COVID-19): a systematic review of imaging findings in 919 patients," American Journal of Roentgenology, vol. 215, no. 1, pp. 87-93, 2020.

[17] T. Fujii, T. Nakamura, and A. Iwamoto, "Pneumocystis pneumonia in patients with HIV infection: clinical manifestations, laboratory findings, and radiological features," Journal of Infection and Chemotherapy, vol. 13, no. 1, pp. 1-7, 2007.

[18] C. Hani, N. H. Trieu, I. Saab et al., "COVID-19 pneumonia: a review of typical CT findings and differential diagnosis," Diagn Interv Imaging, vol. 101, no. 5, pp. 263-268, 2020.

[19] M. Ackermann, S. E. Verleden, M. Kuehnel et al., "Pulmonary vascular endothelialitis, thrombosis, and angiogenesis in COVID-19," The New England Journal of Medicine, vol. 383, no. 2, pp. 120-128, 2020.

[20] T. Menter, J. D. Haslbauer, R. Nienhold et al., "Postmortem examination of COVID-19 patients reveals diffuse alveolar damage with severe capillary congestion and variegated findings in lungs and other organs suggesting vascular dysfunction," Histopathology, vol. 77, no. 2, pp. 198-209, 2020.

[21] J. M. Connors and J. H. Levy, "Thromboinflammation and the hypercoagulability of COVID-19," Journal of Thrombosis and Haemostasis, vol. 18, no. 7, pp. 1559-1561, 2020.

[22] https://ijisrt.com/assets/upload/files/IJISRT20JUL095.pdf\% 20.

[23] Eli Lilly and Company, Fact Sheet for Healthcare Providers: Emergency Use Authorization (EUA) of Baricitinib, Eli Lilly and Company, Indianapolis, IN, USA, 2020, https://www.fda. gov/media/143823/download.

[24] National Institutes of Health, Coronavirus Disease 2019 (COVID-19) Treatment Guidelines, National Institutes of Health, Bethesda, MD, USA, 2021, https://covid19treatmentguidelines. nih.gov/.

[25] IDSA, Infectious Diseases Society of America Guidelines on the Treatment and Management of Patients with COVID-19, IDSA, Arlington, VA, USA, 2020, https://www.idsociety.org/ practice-guideline/covid-19-guideline-treatment-and-manag ement.

[26] UpToDate, Treatment-and-Prevention-of-Pneumocystis-Infection-In-Patients-with-HIV, UpToDate, Waltham, MA, USA, 2021, https://www.uptodate.com/contents/treatmentand-prevention-of-pneumocystis-infection-in-patients-withhiv.

[27] J. A. C. Sterne, S. Murthy, J. V. Diaz et al., "Association between administration of systemic corticosteroids and mortality among critically ill patients with COVID-19: a meta-analysis," JAMA, vol. 324, no. 13, pp. 1330-1341, 2020.
[28] R. A. Siemieniuk, J. J. Bartoszko, L. Ge et al., "Drug treatments for COVID-19: living systematic review and network metaanalysis," BMJ, vol. 370, Article ID m2980, 2020.

[29] P. Horby, W. S. Lim, L. Linsell et al., "Dexamethasone in hospitalized patients with COVID-19," The New England Journal of Medicine, vol. 384, no. 8, pp. 693-704, 2021.

[30] National Institutes of Health-University of California, "Consensus statement on the use of corticosteroids as adjunctive therapy for pneumocystis pneumonia in the acquired immunodeficiency syndrome," The New England Journal of Medicine, vol. 323, no. 21, pp. 1500-1504, 1990.

[31] Available at: https://clinicalinfo.hiv.gov/en/guidelines/adultand-adolescent-opportunistic-infection/whats-new-guidelines. 